\title{
Carbon monoxide in the solar atmosphere
}

\section{Radiative cooling by $\mathrm{CO}$ lines}

\author{
S. Wedemeyer-Böhm ${ }^{1,3}$ and M. Steffen ${ }^{2}$ \\ 1 Kiepenheuer-Institut für Sonnenphysik, Schöneckstraße 6, 79104 Freiburg, Germany \\ 2 Astrophysikalisches Institut Potsdam, An der Sternwarte 16, 14482 Potsdam, Germany \\ e-mail: msteffen@aip.de \\ 3 Institute of Theoretical Astrophysics, University of Oslo, PO Box 1029 Blindern, 0315 Oslo, Norway
}

Received 3 August 2006 / Accepted 6 December 2006

\section{ABSTRACT}

\begin{abstract}
Aims. The role of carbon monoxide as a cooling agent for the thermal structure of the mid-photospheric to low-chromospheric layers of the solar atmosphere in internetwork regions is investigated.

Methods. The treatment of radiative cooling via spectral lines of carbon monoxide (CO) has been added to the radiation chemohydrodynamics code CO5BOLD. The radiation transport has now been solved in a continuum band with Rosseland mean opacity and an additional band with CO opacity. The latter is calculated as a Planck mean over the CO band between 4.4 and $6.2 \mu \mathrm{m}$. The time-dependent $\mathrm{CO}$ number density is derived from the solution of a chemical reaction network.

Results. The CO opacity indeed causes additional cooling at the fronts of propagating shock waves in the chromosphere. There, the time-dependent approach results in a higher CO number density compared to the equilibrium case and hence in a larger net radiative cooling rate. The average gas temperature stratification of the model atmosphere, however, is only reduced by roughly $100 \mathrm{~K}$. Also the temperature fluctuations and the $\mathrm{CO}$ number density are only affected to small extent. A numerical experiment without dynamics shows that the $\mathrm{CO}$ cooling process works in principle and drives the atmosphere to a cool radiative equilibrium state. At chromospheric heights, the radiative relaxation of the atmosphere to a cool state takes several $1000 \mathrm{~s}$. The CO cooling process thus would seem to be too slow compared to atmospheric dynamics to be responsible for the very cool temperature regions observed in the solar atmosphere. Conclusions. The hydrodynamical timescales in our solar atmosphere model are much too short to allow for the radiative relaxation to a cool state, thus suppressing the potential thermal instability due to carbon monoxide as a cooling agent. Apparently, the thermal structure and dynamics of the outer model atmosphere are instead determined primarily by shock waves.
\end{abstract}

Key words. Sun: chromosphere - Sun: photosphere - hydrodynamics - radiative transfer - astrochemistry

\section{Introduction}

Carbon monoxide (CO) has been observed in the solar atmosphere (e.g., Noyes \& Hall 1972; Ayres 1981; Ayres et al. 1986; Solanki et al. 1994; Uitenbroek et al. 1994) for more than 30 years now. The discovery of very low temperatures in extreme limb observations of strong CO lines at $5 \mu \mathrm{m}$ by Noyes $\&$ Hall provoked a crisis in our understanding of the thermal structure of the outer layers of the Sun and solar-type stars (Johnson 1973; Wiedemann et al. 1994), since the very existence of $\mathrm{CO}$ molecules in significant amounts requires much lower gas temperatures than predicted by standard semi-empirical models (Vernazza et al. 1981; Fontenla et al. 1993). Due to its infrared emission, carbon monoxide is known to be a strong cooling agent that can actively affect the thermal state of the atmospheric gas itself. Therefore, the idea that $\mathrm{CO}$ was capable of inducing a thermal bifurcation of the solar atmosphere has attracted considerable attention during the past three decades (cf. Ayres 1981; Kneer 1983; Muchmore \& Ulmschneider 1985; Muchmore 1986; Steffen \& Muchmore 1988; Anderson 1989).

Carbon monoxide molecules can form in significant amounts under the conditions of the solar photosphere and chromosphere. At a given gas density, the equilibrium $\mathrm{CO}$ concentration is a function of temperature, such that the molecules are quickly dissociated towards higher temperatures. Spectral lines of $\mathrm{CO}$ at infrared wavelengths can emit radiation effectively and thus cool the gas, causing a cooling instability. In the absence of compensating mechanical heating, a small perturbation towards lower temperature would already allow the formation of more CO molecules whose infrared lines would further cool the gas and thus induce the formation of even more $\mathrm{CO}$, ultimately leading to a "cooling catastrophe" (cf. Ayres 1981). This way CO can force a cool equilibrium temperature in regions where radiative equilibrium (RE) conditions prevail, whereas a critical amount of mechanical heating can cause a transition to a hot state (e.g., Anderson \& Athay 1989, hereafter AA89).

In the case of the Sun, the consequences of the CO cooling instability have been investigated by a simplified timedependent approach (Steffen \& Muchmore 1988; Muchmore \& Ulmschneider 1985; Muchmore et al. 1988) that treated CO in instantaneous chemical equilibrium (ICE). The ICE assumption was also made by Uitenbroek (2000) for computing the spatial distribution of $\mathrm{CO}$ in a two-dimensional slice from a 3D radiation hydrodynamics simulation. However, this assumption is only valid as long as the timescale governing the formation of carbon monoxide is short compared to the relevant dynamical time scales. A time-dependent treatment was presented by Asensio Ramos et al. (2003), but their simulation was restricted to one spatial dimension in order to keep the problem computationally tractable. 


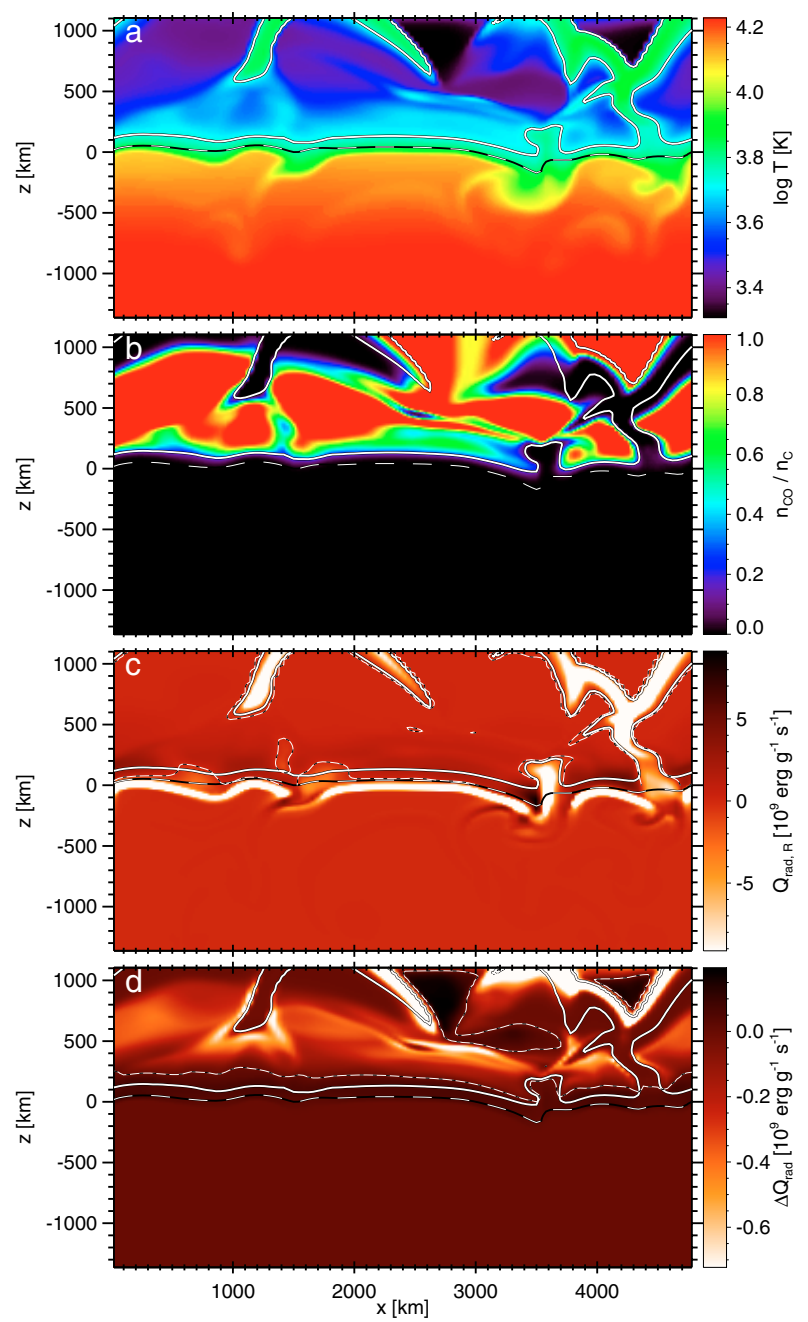

Fig. 1. Two-dimensional slices for an exemplary time step at $t=70390 \mathrm{~s}:$ a) $\operatorname{logarithmic}$ gas temperature $\log T$, b) fraction of carbon atoms bound in $\mathrm{CO} n_{\mathrm{CO}} / n_{\mathrm{C}, \text { total }}$, c) net radiative heating rate $Q_{\mathrm{rad}, \mathrm{R}}$ for the simulation without CO line cooling, and d) the relative difference $\Delta Q_{\mathrm{rad}}$ between the runs with and without $\mathrm{CO}$ cooling. Note that the colour range has been limited in the last panel in order to to make smaller variations visible. The full range is $\Delta Q_{\mathrm{rad}}=[-2.54,0.20] \times 10^{9} \mathrm{erg} \mathrm{g}^{-1} \mathrm{~s}^{-1}$. The lines represent contours for $T=5000 \mathrm{~K}$ (white solid) and Rosseland optical depth unity (long-dashed). The short-dashed line in panels c and d marks $Q_{\mathrm{rad}, \mathrm{R}}=0$ and $\Delta Q_{\mathrm{rad}}=0$, respectively.

Recently, detailed 2D simulations of the distribution of carbon monoxide in the solar atmosphere (Wedemeyer-Böhm et al. 2005, hereafter Paper I) were carried out with the radiation hydrodynamics code $\mathrm{CO}^{5}$ BOLD (Freytag et al. 2002; Wedemeyer et al. 2004), which can treat chemical reaction networks timedependently. It was shown that the bulk of CO is located in cooler regions in the middle photosphere and that a large fraction of all carbon atoms is bound in carbon monoxide in the layers above, with the exception of hot propagating shock waves (see Fig. 1). In contrast to the ICE approach, the high shock temperatures do not cause instantaneous destruction of $\mathrm{CO}$ but rather gradual dissociation of the molecules on chemical timescales. Clearly, the highly dynamic and intermittent nature of the solar chromosphere makes the time-dependent approach mandatory for studying the influence of $\mathrm{CO}$ cooling.

The new two-dimensional simulations presented here were done with an upgraded code version that now accounts for radiative cooling by $\mathrm{CO}$ lines, using the simplified treatment by
Steffen \& Muchmore (1988, hereafter SM88) and Muchmore \& Ulmschneider (1985, hereafter MU85). We describe the method and the new simulations in Sects. 2 and 3, respectively, and present the results in Sect. 4, followed by discussion and conclusions in Sect. 5.

\section{Method}

The applied radiation chemo-hydrodynamics code is described in more detail in Paper I. The only difference to the simulations presented in Paper I concerns the radiative transfer. Instead of the grey, i.e., frequency-independent, approach we use two frequency bands as described by SM88. The first band uses the grey Rosseland opacity $\kappa_{\mathrm{R}}$ constructed from OPAL/PHOENIX data (Iglesias et al. 1992; Hauschildt et al. 1997). It excludes the wavelength region around the $\mathrm{CO}$ fundamental vibrationrotation band in the infra-red beyond $4.6 \mu \mathrm{m}$ that is accounted for in the second band. The opacity in the second band consists of the grey Rosseland opacity $\kappa_{\mathrm{R}}$ and an additional Planck mean $\mathrm{CO}$ opacity $\kappa_{\mathrm{CO}}$ as a function of gas temperature and $\mathrm{CO}$ number density. The latter results from the preceding solution of the chemical reaction network (see Paper I).

\section{Simulation}

The same initial model as in Paper I is used so that the simulations with and without $\mathrm{CO}$ cooling can be compared directly. The model consists of 120 horizontal by 140 vertical grid cells with a total extent of $4800 \mathrm{~km}$ by $2500 \mathrm{~km}$. It describes a small portion of the solar surface layers, just large enough to fit in a few granulation cells. The chemical composition is the same for each cell in the initial model. The simulation was advanced for $86000 \mathrm{~s}$ of solar time. The results presented here only refer to the last $50000 \mathrm{~s}$, whereas the first $36000 \mathrm{~s}$ are reserved as a relaxation phase to ensure the decay of possible initial perturbations. The lower and upper boundaries are located at heights $z=-1479 \mathrm{~km}$ and $z=1021 \mathrm{~km}$, respectively, where the origin $z=0 \mathrm{~km}$ is defined by the average Rosseland optical depth of unity. A corresponding three-dimensional simulation is currently in production (Wedemeyer-Böhm et al., in press).

\section{Results}

\subsection{Net radiative heating rate}

Direct comparison of the two-dimensional simulations with (this paper) and without (Paper I) time-dependent CO opacity shows that inclusion of the spectral band of $\mathrm{CO}$ causes additional cooling at the fronts of propagating shock waves in the chromosphere. There, the time-dependent approach results in a higher $\mathrm{CO}$ number density compared to the equilibrium case and thus in a higher net radiative cooling rate. These small differences make the simulations drift apart so that only a statistical comparison makes sense. However, we illustrate the effect of CO line cooling by comparing the radiative heating rates calculated with and without additional CO line opacity for a given model structure (see Fig. 1). The chosen snapshot is analysed in more detail in Paper I.

For reference, panels a and b of the figure show gas temperature and the fraction of carbon atoms bound in $\mathrm{CO}, n_{\mathrm{CO}} / n_{\mathrm{C} \text {,total }}$, respectively. The latter refers to the total number density of all carbon atoms, which is here the sum of the densities of included species $\mathrm{C}, \mathrm{CO}$, and $\mathrm{CH}$. The solid line is a contour for a temperature of $T=5000 \mathrm{~K}$ that outlines the prominent shock wave 
in the low chromosphere and also a high-temperature structure belonging to the reversed granulation pattern in the middle photosphere.

The net radiative heating rate $Q_{\mathrm{rad}, \mathrm{R}}$ for the simulation without radiative $\mathrm{CO}$ cooling is displayed in panel c. The rate represents the change in the internal energy of the gas per unit time and mass due to radiation. Positive values indicate that energy is added, i.e., the gas is heated, whereas negative values indicate the release of energy via radiative emission, i.e. radiative cooling. For most of the computational domain, $Q_{\text {rad,R }}$ is close to zero or at least small. In contrast, high negative values are found in shock waves and close to optical depth unity (the visible "surface" of the Sun) where strong radiative emission cools the gas. There are also some locations with high positive values, indicating radiative heating of the gas. This is obviously the case for volume elements that are surrounded by high-temperature regions, e.g. at $x=3500 \mathrm{~km}, z=-100 \mathrm{~km}$.

Panel d of Fig. 1 shows the additional contribution of $\mathrm{CO}$ to the net radiative heating rate, $\Delta Q_{\text {rad }}$, which was retrieved by subtracting the rates from the radiative transfer calculation with and without additional $\mathrm{CO}$ opacity $\left(\Delta Q_{\mathrm{rad}}=Q_{\mathrm{rad}, \mathrm{CO}}-Q_{\mathrm{rad}, \mathrm{R}}\right)$. Most obvious is the emission (white) at the fronts of the shock waves where a significant amount of CO can still effectively cool before being dissociated by the approaching hot wave fronts. The effect is much smaller outside shock waves. On the other hand, there are cool regions with a high $\mathrm{CO}$ concentration that absorbs radiation, resulting in a locally enhanced heating of the gas. Although this can make a local difference in the overall contribution of the $\mathrm{CO}$ lines, which are restricted to a relatively narrow wavelength range in the infrared, the overall effect remains minor compared to the continuum contribution.

\subsection{Average stratification}

The comparison of the full sequence duration is done by means of average stratifications as shown in Fig. 2 for gas temperature, root mean square (rms) temperature fluctuation, and $\mathrm{CO}$ number density. The average temperature of the simulation with $\mathrm{CO}$ radiative cooling is a bit lower with a maximum deviation of $-128 \mathrm{~K}$ at a height of $495 \mathrm{~km}$, whereas the average temperature fluctuation is increased by maximum $124 \mathrm{~K}$ at $z=105 \mathrm{~km}$. The higher fluctuations cause a small increase of $0.25 \times 10^{12} \mathrm{~cm}^{-3}(11 \%)$ in average CO number density also at a height of $105 \mathrm{~km}$.

\subsection{Cooling experiment}

The surprisingly small effect of the CO cooling on the thermal structure of the atmosphere can be understood from the results of a numerical experiment. As the strong dynamics in the atmosphere disturb the $\mathrm{CO}$ formation, we run additional simulations in which we damp velocity fluctuations above the middle photosphere by a "drag-force" characterised by a timescale of $\tau_{\text {damp }}=3 \mathrm{~s}$. After each time step $\delta t$, all spatial velocity components $v_{j}$ are reduced with a reduction factor $r$ that ensures a smooth transition between the lower undamped domain $(z \leq$ $\left.z_{1}=130 \mathrm{~km}\right)$ and the upper damped layers $\left(z \geq z_{2}=310 \mathrm{~km}\right)$ :

$$
\begin{aligned}
v_{j}^{\text {new }}\left(i_{x}, i_{z}\right) & =r v_{i}^{\text {old }}\left(i_{x}, i_{z}\right), \forall j \\
r & =1-\frac{\delta t \zeta^{2}(3-2 \zeta)}{\tau_{\text {damp }}}, r \geq 0 \\
\zeta & =\frac{z\left(i_{z}\right)-z_{1}}{z_{2}-z_{1}}, 0 \geq \zeta \geq 1 .
\end{aligned}
$$
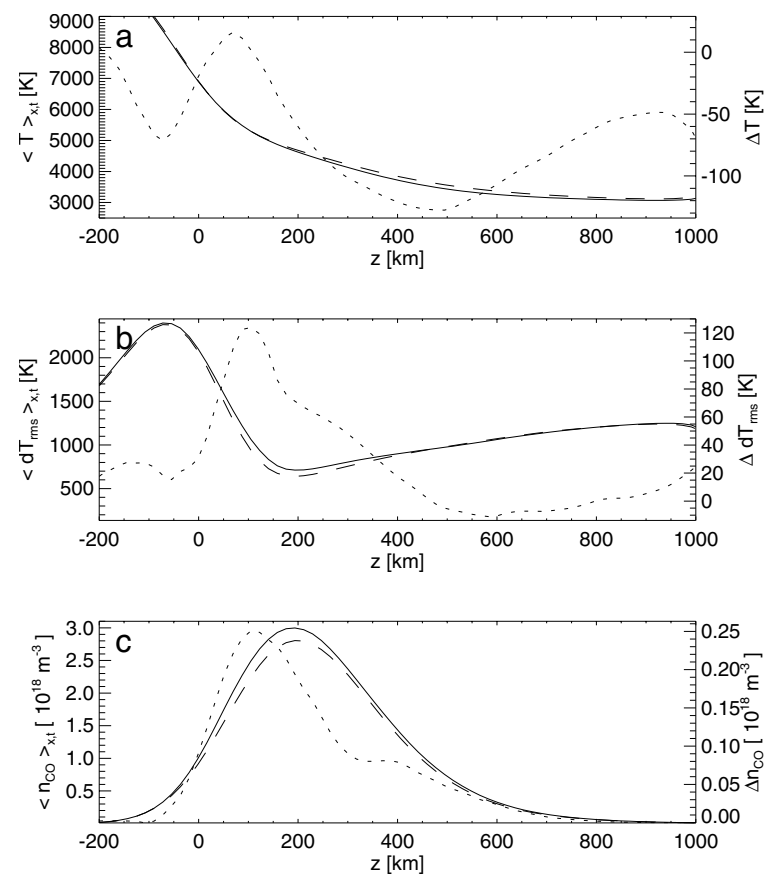

Fig. 2. Horizontally and temporally averaged temperature stratification a), temperature fluctuation b), and $\mathrm{CO}$ number density c) for the simulations with (solid line) and without (dashed line) radiative $\mathrm{CO}$ cooling. The dotted lines represent the difference between both cases, e.g. $\Delta T=\left\langle T_{\mathrm{CO}}\right\rangle_{x, t}-\left\langle T_{\mathrm{R}}\right\rangle_{x, t}$.

This way the convection in the lower part $(\zeta=0)$ remains unaffected $(r=1)$, while the chromosphere $(\zeta=1)$ can relax to hydrostatic, chemical, and radiative equilibrium. The initial models are constructed from the reference model without additional CO cooling (see Paper I). The lower part of the model $\left(z<z_{1}\right)$ is unchanged. Above we smoothly attach a new atmosphere with the gas density set to the horizontal average of the density in the original model and the gas temperature changed to a constant value of $2000 \mathrm{~K}$ or $7000 \mathrm{~K}$ (see Fig. 3a), which are roughly the extreme values encountered in the model chromosphere. The initial abundances of the molecular species including $\mathrm{CO}$ are again set to very small numbers.

Now we run the first simulation with only grey radiative transfer with the mean Rosseland opacity band, i.e. frequencyindependent without $\mathrm{CO}$ band, to derive a reference model in grey radiative equilibrium. The resulting temperature stratification (see black line in Fig. 3a) reaches an equilibrium value of $4680 \mathrm{~K}$ in the upper photosphere and above. The next simulations with the additional $\mathrm{CO}$ band produce the same temperature stratification up to $z \sim 150 \mathrm{~km}$. Above that height, CO line cooling reduces the mean gas temperature significantly. For instance, an equilibrium value of $\sim 3300 \mathrm{~K}$ is reached at a height of $500 \mathrm{~km}$, whereas at $650 \mathrm{~km}$ the final value is close to $2650 \mathrm{~K}$, independent of the initial chromospheric temperature.

The temporal evolution of the average temperature is shown for the chromospheric height of $650 \mathrm{~km}$ in panel b. Even for high initial temperature, the grey radiative equilibrium is reached after only a few minutes. Also the simulation with the cool initial chromosphere $(2000 \mathrm{~K})$ shows a steeper evolution towards the grey $\mathrm{RE}$ at the very beginning, but is dominated by the relaxation towards the CO-driven cool state afterwards. This second part is much slower and approximately exponential with timescales of at least $\sim 1000 \mathrm{~s}$. The total time for thermal relaxation to a cool state is of the order of $3000 \mathrm{~s}$. The hot initial chromosphere 

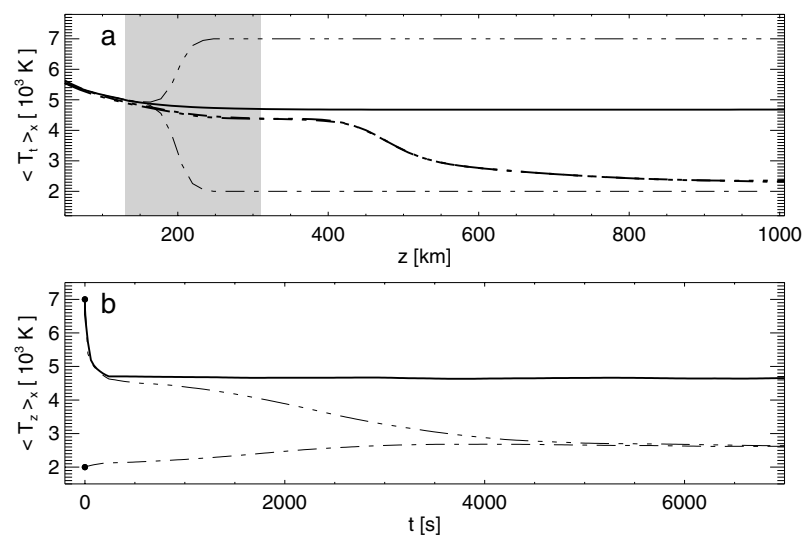

Fig. 3. Time evolution towards radiative equilibrium (RE) for simulations without (solid) and with $\mathrm{CO}$ line cooling for different initial chromospheric temperatures of $2000 \mathrm{~K}$ (dot-dashed) and $7000 \mathrm{~K}$ (tripledot-dashed): a) horizontally averaged temperature stratification for initial time step (thin) and after $10000 \mathrm{~s}$ (thick) and b) time evolution of the horizontally averaged temperature at $z=650 \mathrm{~km}$. The shaded area marks the transition layer for artificial damping of velocity fluctuations.

evolves even slower towards the equilibrium state. In all cases the relaxation timescales are long compared to the hydrodynamical timescales present in the simulations.

\subsection{Prescribed mechanical heating}

We extended the cooling experiment (see Sect. 4.3) and added a prescribed (mechanical) heating as done by AA89 (cf. their Eq. (1)). The additional heating $Q_{\text {add }}[\mathrm{erg} / \mathrm{g} / \mathrm{s}]$ is a function of column mass density $m$ :

$Q_{\text {add }}(m)=\mu^{-1} q_{0}\left(m / m_{1}\right)^{x} \mathrm{e}^{-m / m_{0}}$,

where $q_{0}$ prescribes the heating rate, $m_{0}=m_{1}=0.0114$ and $x=$ -0.75 . The mean atomic weight $\mu$ is set to $2.167 \times 10^{-24} \mathrm{~g} /$ atom. The heating term is added to the internal energy $\varepsilon$ for each grid cell for each time step of duration $\delta t$ :

$\varepsilon^{\text {new }}\left(i_{x}, i_{z}\right)=\varepsilon^{\text {old }}\left(i_{x}, i_{z}\right)+\delta t Q_{\text {add }}\left(i_{x}, i_{z}\right)$.

The initial model is the same as for the simulation described in Sect. 3. The calculations are carried out until the temperature at all heights reaches equilibrium. The resulting temperature stratifications are shown in Fig. 4 for different values of $q_{0}$. For comparison the non-LTE models by AA89 are plotted. We find the same qualitative dependence of equilibrium temperature stratification on the amount of prescribed heating. Our models have a "temperature minimum" in the upper photosphere/low chromosphere and a steep temperature jump above. Nevertheless there are quantitative differences: (i) our minimum temperatures and also the stratifications for no additional heating are cooler than those of AA89; (ii) the temperature jump occurs deeper in the atmosphere than for AA89; and (iii) the amount of heating required for compensating the $\mathrm{CO}$ cooling is higher compared to AA89. In their calculations the otherwise present cool atmospheric layer cannot form once the heating exceeds a critical value that lies between $\log q_{0}=-16.0$ and -15.5 (see their Fig. 1). In contrast, we still get a temperature minimum with $\log q_{0}=-15.5$, suggesting that the $\mathrm{CO}$ cooling to be compensated is higher in our models. The different height dependence of the average temperature profile is most likely due to differences in the treatment of radiative transfer.

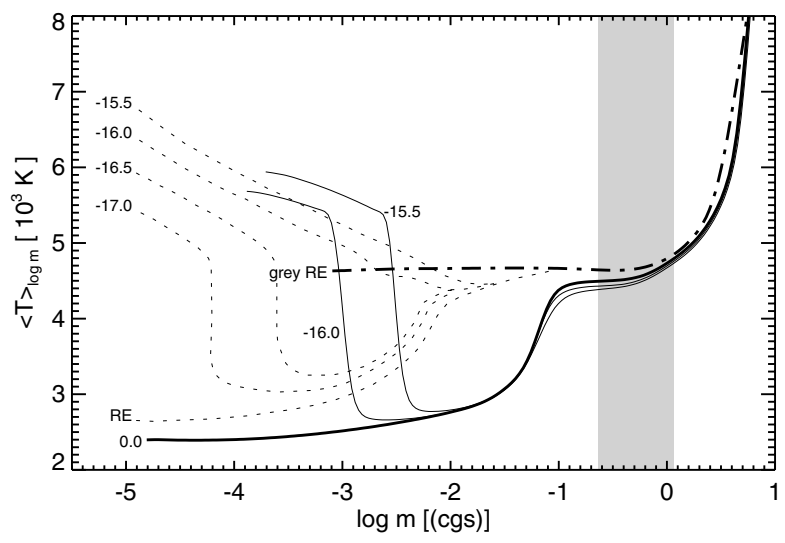

Fig. 4. Cooling experiment with prescribed (mechanical) heating. Gas temperature after $4 \mathrm{~h}$ averaged on column mass scale for different amounts of prescribed (mechanical) heating with $\log q_{0}=-15.5$ and -16.0 (thin solid) and without additional heating (thick solid). The thick dot-dashed line represents a simulation with grey radiative transfer alone (i.e. without CO). The results of AA89 (see their Fig. 1) are plotted for comparison (dotted lines). The corresponding mechanical heating $\left(\log q_{0}\right)$ is noted on the left. The curve annotated with "RE" is the non-LTE RE stratification by Anderson (1989). The shaded area marks the transition layer for artificial damping of velocity fluctuations.

\section{Discussion and conclusions}

Our cooling experiment produces a total time for thermal relaxation to a cool state of the order of $3000 \mathrm{~s}$ at low-chromospheric heights. This result is in line with the earlier results from SM88. The final temperature, however, remains $\sim 350 \mathrm{~K}$ higher in our experiment. This can be partly explained by the use of a detailed chemical reaction network instead of equilibrium $\mathrm{CO}$ densities and the corresponding uncertainties in chemical input data in both cases. Remaining differences can be attributed to the fact that the simulations by Steffen \& Muchmore include a stationary flow that differs from the flow field in our simulations and the resulting amount of additional cooling due to adiabatic expansion. Our cool equilibrium temperature agrees well with the model atmospheres in radiative and hydrostatic equilibrium by Anderson (1989) in which CO cooling reduces the temperature to $2640 \mathrm{~K}$. It is also in line with AA89, who, depending on the amount of prescribed mechanical heating, find temperatures down to $\sim 3000 \mathrm{~K}$, and the $2900 \mathrm{~K}$ already found by Ayres (1981).

The treatment of radiative transfer needs to be simplified in order to keep the problem computationally tractable in the framework of a multi-D radiation chemo-hydrodynamics simulation. The use of only two opacity bands is indeed a strong simplification, in particular in view of the large number of spectral lines of different line strengths in the $\mathrm{CO}$ fundamental vibrationrotational bands. Nordlund (1985) states that "the cooling in the infrared $\mathrm{CO}$ lines is compensated for by a heating in the ODFs in the blue and near UV" if UV opacities $(\lambda<400 \mathrm{~nm})$ are treated as pure absorption, as in the method applied here (based on MU85). The alternative assumption of pure scattering is questionable (cf. Anderson 1989). The fact that we still find a net cooling due to $\mathrm{CO}$ in the absence of atmospheric dynamics can be interpreted such that our simplified LTE treatment overestimates the cooling ability of the IR CO lines. Muchmore et al. (1988) compare the scheme by MU85 with a more detailed scheme with more frequency points and conclude that (i) the $\mathrm{CO}$ overtone bands at $2.2 \mu \mathrm{m}$ play only a minor role compared to the fundamental bands in the $5 \mu \mathrm{m}$ region and (ii) the $\mathrm{CO}$ cooling 
might be overestimated by a factor of three with this simple scheme.

A similar conclusion can be drawn from the comparison of the temperature stratifications from our extended cooling experiment with the NLTE models by AA89 (see Sect. 4.4). It reveals that the equilibrium temperature in the atmosphere is lower than found by AA89 and that the amount of additional heating required to compensate $\mathrm{CO}$ cooling is higher for our models. Obviously, our approach represents an upper limit for the net radiative cooling rate. So if even an over-efficient $\mathrm{CO}$ cooling mechanism cannot induce significant effects on the atmospheric stratification, as shown in the study presented here, then we can safely conclude that a more realistic treatment with smaller net cooling will have even less effect.

The inclusion of CO line opacity in our simulations indeed produced additional radiative cooling at the front of fastpropagating shock waves in the upper atmospheric layers due to a higher $\mathrm{CO}$ number density compared to the instantaneous chemical equilibrium (ICE) case. In contrast to the ICE case, the dissociation of $\mathrm{CO}$ proceeds on a finite timescale so that the $\mathrm{CO}$ number density in the front of a passing shock wave is reduced gradually and not instantaneously. As a consequence, additional radiative cooling is present at these locations. The resulting changes in the thermal structure, however, remain small.

It is commonly known that the dynamics tend to be too strong in $2 \mathrm{D}$ compared to $3 \mathrm{D}$. But even in our 3D simulations we do not find the passage of shock waves to be less frequent, and the temperature fluctuations are comparable in amplitude except for somewhat larger differences in the middle photosphere (Wedemeyer 2003). The 3D model by Wedemeyer-Böhm et al. (in press) nevertheless yields a $\mathrm{CO}$ distribution that is very similar to the 2D results presented here, confirming that the dynamics in the 2D model are reasonably realistic.

We conclude that the self-amplifying cooling process of $\mathrm{CO}$ can in principle operate in the solar atmosphere (as demonstrated in Fig. 3 and by earlier works) and could lead to a bifurcation between cool areas dominated by RE and warmer areas dominated by mechanical heating. However, a relaxation to a cool state of the solar atmosphere is prevented in the presence of pronounced atmospheric dynamics as predicted by our models. The crucial point is that the $\mathrm{CO}$ radiative relaxation timescales are too long compared to the frequent passage of shock waves. Instead, the co-existence of hot and cool regions is predominantly caused by mechanical heating due to these propagating shock waves and adiabatic cooling of the resulting post-shock regions (Wedemeyer et al. 2004). Without any doubt, the present state-of-the-art simulations still admit to some limitations, and the predictions of the velocity and temperature fluctuations in the higher layers are thus affected by uncertainties. Detailed observational tests of atmospheric properties, such as temporal and spatial temperature and velocity variations and their centre-tolimb behaviour, are therefore highly desired.

Acknowledgements. We would like to thank T. Ayres, J. Bruls, O. Steiner, and R. J. Rutten for discussion and helpful comments. S.W.B. is grateful to the Institut für Theoretische Physik und Astrophysik der Universität Kiel for hospitality. This work was supported by the Deutsche Forschungsgemeinschaft (DFG), project Ste 615/5.

\section{References}

Anderson, L. S. 1989, ApJ, 339, 558

Anderson, L. S., \& Athay, R. G. 1989, ApJ, 346, 1010 (AA89)

Asensio Ramos, A., Trujillo Bueno, J., Carlsson, M., \& Cernicharo, J. 2003, ApJ, 588, L61

Ayres, T. R. 1981, ApJ, 244, 1064

Ayres, T. R., Testerman, L., \& Brault, J. W. 1986, ApJ, 304, 542

Ayres, T. R., Plymate, C., \& Keller, C. U. 2006, ApJS, 165, 618

Fontenla, J. M., Avrett, E. H., \& Loeser, R. 1993, ApJ, 406, 319

Freytag, B., Steffen, M., \& Dorch, B. 2002, Astron. Nachr., 323, 213

Hauschildt, P. H., Baron, E., \& Allard, F. 1997, ApJ, 483, 390

Iglesias, C. A., Rogers, F. J., \& Wilson, B. G. 1992, ApJ, 397, 717

Johnson, H. R. 1973, ApJ, 180, 81

Kneer, F. 1983, A\&A, 128, 311

Muchmore, D. 1986, A\&A, 155, 172

Muchmore, D., \& Ulmschneider, P. 1985, A\&A, 142, 393 (MU85)

Muchmore, D., Ulmschneider, P., \& Kurucz, R. L. 1988, A\&A, 201, 138

Nordlund, A. 1985, in Theo. Prob. High Res. Solar Phys., ed. H. U. Schmidt, 1

Noyes, R. W., \& Hall, D. N. B. 1972, ApJ, 176, L89

Solanki, S. K., Livingston, W., \& Ayres, T. 1994, Science, 263, 64

Steffen, M., \& Muchmore, D. 1988, A\&A, 193, 281 (SM88)

Uitenbroek, H. 2000, ApJ, 531, 571

Uitenbroek, H., Noyes, R. W., \& Rabin, D. 1994, ApJ, 432, L67

Vernazza, J. E., Avrett, E. H., \& Loeser, R. 1981, ApJS, 45, 635

Wedemeyer, S. 2003, Ph.D. Thesis, Univ. Kiel,

http://e-diss.uni-kiel.de/diss_764/

Wedemeyer, S., Freytag, B., Steffen, M., Ludwig, H.-G., \& Holweger, H. 2004, A\&A, 414, 1121

Wedemeyer-Böhm, S., Kamp, I., Bruls, J., \& Freytag, B. 2005, A\&A, 438, 1043 (Paper I)

Wedemeyer-Böhm, S., Kamp, I., Freytag, B., Bruls, J., \& Steffen, M., in NSO

Workshop No. 23, ed. H. Uitenbroek, \& J. Leibacher 2007, in press

Wiedemann, G., Ayres, T. R., Jennings, D. E., \& Saar, S. H. 1994, ApJ, 423, 806 